\title{
Intra-operative optical coherence tomography in glaucoma surgery-a systematic review
}

\author{
Bryan C. H. Ang ${ }^{1} \cdot$ Sheng Yang Lim $\mathbb{1}^{2} \cdot$ Syril Dorairaj $^{3}$
}

Received: 6 July 2019 / Revised: 1 October 2019 / Accepted: 4 November 2019 / Published online: 26 November 2019

(c) The Author(s), under exclusive licence to The Royal College of Ophthalmologists 2019

\begin{abstract}
The application of the OCT in clinical ophthalmology has expanded significantly since its introduction more than 20 years ago. There has been recent growing interest in the application of intra-operative optical coherence tomography (iOCT). The iOCT's ability to enhance visualisation and depth appreciation has the potential to be further exploited in glaucoma surgery, especially with the emergence of Minimally Invasive Glaucoma Surgery (MIGS) - to facilitate targeted device placement and fine surgical manoeuvres in the angles, the sub-conjunctival layer and the suprachoroidal space. Hence, this study aims to appraise the current literature on the applications of iOCT in glaucoma surgery. A total of 79 studies were identified following a literature search adhering to PRISMA guidelines. After full text evaluation, 10 studies discussing iOCT use in glaucoma surgery were included. Traditional glaucoma filtering procedures reviewed included trabeculectomy surgery, goniosynechiolysis, bleb needling and glaucoma drainage device implantation. MIGS procedures reviewed included canaloplasty, trabecular aspiration, ab-interno trabectome and the XEN45 gel stent. iOCT use in ophthalmic surgery is becoming increasingly prevalent and has already been applied in various surgeries and procedures in the field of glaucoma. With the greater adoption of MIGS, iOCT may further contribute in facilitating surgical techniques and improving outcomes. While iOCT offers many advantages, there are still limitations to be overcome-iOCT technology continues to evolve to optimise imaging quality and user-experience.
\end{abstract}

\section{Introduction}

The application of the optical coherence tomography (OCT) in clinical ophthalmology has expanded significantly since its introduction more than 20 years ago [1]. In particular, there has been growing interest in the application of intra-operative OCT (iOCT) in ophthalmic surgery. Microscope-integrated platforms which demonstrate superior usability and operability compared with their portable counterparts of the past,

These authors contributed equally: BCH Ang, SY Lim

Syril Dorairaj

dorairaj.syril@mayo.edu

1 Department of Ophthalmology, Tan Tock Seng Hospital, National Healthcare Group Eye Institute, Singapore, Singapore

2 Yong Loo Lin School of Medicine, National University of Singapore, Singapore, Singapore

3 Department of Ophthalmology, Mayo Clinic, Jacksonville, Florida, USA are now FDA-approved for clinical use. Over the last decade, the iOCT has been successfully introduced into cataract, vitreoretinal, corneal and anterior segment surgeries [2-12].

In glaucoma, the application of the iOCT has also been reported in various studies and reports [7, 8, 13-29], in traditional glaucoma filtering surgery (trabeculectomy and glaucoma drainage device implantation), goniosynechiolysis, bleb needling and canaloplasty. This paper aims to, through a systemic review, consolidate data from available literature describing the existing and potential roles of the iOCT in glaucoma surgeries and procedures. Potential further applications of the iOCT are also discussed, particularly in view of the emergence of Minimally Invasive Glaucoma Surgery (MIGS). Finally, the current limitations and recent advancements in iOCT technology are summarised.

\section{Methods}

A comprehensive search of MEDLINE, EMBASE, Current Contents, Cochrane Central Register of Controlled Trials (CENTRAL) databases was performed on 29th March 
2019. A combination of subject headings and text words was used as needed to define iOCT and glaucoma procedures. We employed the terms '((Intra-operative optical coherence tomography) OR iOCT) AND (trabeculotomy OR canaloplasty OR trabecular aspiration OR stent OR istent OR hydrus OR itrack OR abic OR (ab interno canaloplasty) OR cypass OR microshunt OR innfocus OR xen OR aquesys OR allergan OR trabectome OR neomedix OR trabeculectomy OR goniosynechiolysis OR bleb OR glaucoma drainage device* OR glaucoma OR (minimally AND invasive AND glaucoma AND surgery))'. The search workflow was designed in adherence to the preferred reporting items for systematic reviews and meta-analyses (PRISMA) statement [30] (Fig. 1).

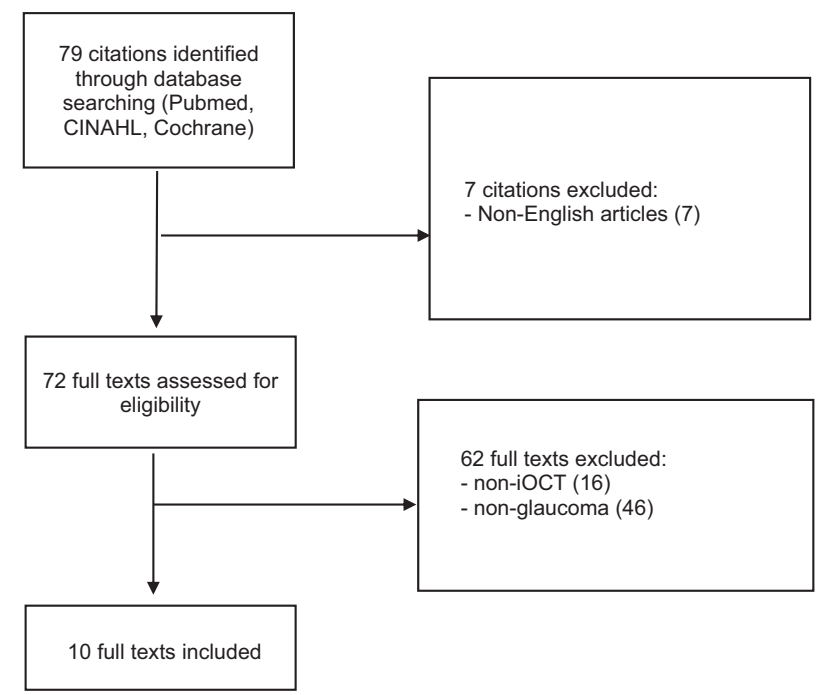

Fig. 1 PRISMA flowchart
All identified studies were assessed independently by two authors (SY and $\mathrm{BCH}$ ) to determine eligibility for inclusion in the analysis. Articles not written in English were excluded from the study. There was no limit on the type of study or minimum patient number required. Cadaveric studies were included in this systematic review.

\section{Results}

A total of 79 studies were identified following a literature search following PRISMA guidelines. After full text evaluation, ten studies discussing iOCT use in glaucoma surgery were included [15, 18, 21, 22, 27-29, 31-33]. Various glaucoma surgical techniques were reported on, in addition to the role of heads-up display (HUD) in iOCT use and iOCT-compatible instruments. Four cadaveric eyes, 30 patients with conditions including but not limited to primary open-angle glaucoma, pigment dispersion, pseudoexfoliation, steroid-induced, uveitic, mixed mechanism glaucoma, as well as feedback from 20 surgeons were included. A summary of each study design, population and patient characteristics (if any) is shown in Table 1.

The use of the iOCT has been described in the following glaucoma procedures.

\section{Trabeculectomy}

Trabeculectomy is a commonly performed glaucoma filtration surgery which aims to reduce intraocular pressure by creating a partial thickness scleral flap to allow drainage of aqueous humour to the sub-conjunctival space. A good scleral flap creation is a critical step in this surgery, with the

Table 1 Studies included

\begin{tabular}{|c|c|c|c|c|}
\hline Author, year & Type of study & Intervention & $\begin{array}{l}\text { No. of Eyes/ } \\
\text { Patients }\end{array}$ & Patient characteristics \\
\hline Palacios 2019 & $\begin{array}{l}\text { Experimental and } \\
\text { clinical study }\end{array}$ & $\begin{array}{l}\text { Heads up display for iOCT } \\
\text { for } 20 \text { surgeons }\end{array}$ & 0 & NA \\
\hline Swaminathan 2017 & Cadaveric study & 3 Glaucoma drainage device & 3 eyes & NA \\
\hline Junker 2017 & Retrospective case series & 9 Trabectome & 9 patients & $\begin{array}{l}\text { Patients with primary open-angle glaucoma, pigment } \\
\text { dispersion glaucoma or pseudoexfoliation glaucoma }\end{array}$ \\
\hline Tanito 2017 & Prospective case series & 9 Trabeculotomy & 9 eyes, 9 patients & $\begin{array}{l}5 \text { primary open-angle glaucoma, } 1 \text { pseudoexfoliation } \\
\text { glaucoma, } 1 \text { steroid induced glaucoma, } 1 \text { uveitic glaucoma, } \\
1 \text { mixed mechanism glaucoma }\end{array}$ \\
\hline Ehlers 2017 & Experimental study & iOCT compatible instruments & 1 eye & Cadaveric eyes \\
\hline Dada 2016 & Prospective case series & 2 Bleb needling & 2 patients, 2 eyes & $\begin{array}{l}1 \text { flat and vascularised bleb } 10 \text { weeks post-operatively, } \\
1 \text { encapsulated bleb } 3 \text { months post-operatively }\end{array}$ \\
\hline Siebelmann 2016 & Retrospective case report & 1 Canaloplasty & 1 patient, 1 eye & 1 primary open-angle glaucoma \\
\hline Kumar 2015 & Prospective case series & $\begin{array}{l}1 \text { phaco-trabeculectomy, } \\
1 \text { AGV implantation, } \\
1 \text { goniosynechiolysis, } 1 \text { bleb } \\
\text { needling }\end{array}$ & 4 patients, 4 eyes & $\begin{array}{l}1 \text { medically uncontrolled primary open-angle glaucoma with a } \\
\text { visually significant cataract, } 1 \text { intractable glaucoma following a } \\
\text { failed penetrating keratoplasty, } 1 \text { failing trabeculectomy bleb }\end{array}$ \\
\hline Heindl 2014 & Prospective case series & $\begin{array}{l}1 \text { trabecular aspiration } \\
1 \text { trabeculotomy }\end{array}$ & 2 patients, 2 eyes & $\begin{array}{l}1 \text { pseudoexfoliation glaucoma combined with phaco trabecular } \\
\text { aspiration } \\
1 \text { primary open-angle glaucoma }\end{array}$ \\
\hline Geerling 2005 & Prospective case series & $\begin{array}{l}1 \text { trabeculectomy } \\
3 \text { lamellar keratoplasty }\end{array}$ & 4 patients & NIL \\
\hline
\end{tabular}


depth of incision, regularity and thickness of the scleral flap potentially influencing success rates of the surgery [34]. The iOCT allows the trabeculectomy surgeon to ascertain the depth of the initial scleral incision, as well as to visualise the regularity of the dissection and thickness of the scleral flap. While the current state of technology does not allow precise depth identification, iOCT changes the nature of the procedure from a 'blind' approach to a 'visually guided' approach. This is particularly important in eyes with high axial myopia, where the thin sclera increases the risk of full-thickness dissection as well as subsequent scarring [34], contributing to a higher rate of failure [35]. The iOCT also allows more accurate visualisation of the sclerostomy and iridectomy during trabeculectomy [22]. Beyond achieving better surgical outcomes, iOCT may also be used as a tool for training residents to perform glaucoma filtering surgery [36].

\section{Goniosynechiolysis}

Goniosynechiolysis involves the mechanical release of peripheral anterior synechiae (PAS). This is usually performed with the aid of intra-operative gonioscopy and an instrument such as a cyclodialysis spatula to widen the irido-corneal angle. Trauma or misdirected force at the angle during the procedure can result in iridodialysis or haemorrhage.

However, intra-operative gonioscopy can be challenging, particularly for beginning angle surgeons. The iOCT allows the surgeon to perform goniosynechiolysis without the use of intra-operative gonioscopy. Post-synechiolysis, the iOCT may also enable the surgeon to more objectively assess the adequacy of synechiolysis and the effectiveness of the procedure in opening the angle [22]. However, in eyes with hazy corneas, the iOCT may be of limited advantage, given that it shares similar limitations as a table-top SDOCT in these scenarios.

\section{Bleb needling}

Post-trabeculectomy bleb encapsulation has been reported in $13-29 \%$ of patients, with bleb needling required in $20 \%$ of cases [22]. This procedure is performed either in the outpatient clinic setting or in the operating theatre, often with the use of antimetabolites. This procedure aims to restore aqueous filtration into the sub-conjunctival space by mechanically breaking the connective capsule under the bleb wall and releasing fibrotic sub-conjunctival and subtenon's adhesions [25].

Unfortunately, this being a "blind" procedure, the formation of a visible, diffuse bleb becomes the only indicator of success. This procedure may also be complicated by subconjunctival haemorrhage, which obscures the surgeon's view and compromises the effectiveness of needling. The iOCT may be useful in bleb needling by allowing the visualisation of deeper tissue and structures within the bleb, demonstrating the extent and location of adhesions and loculations [15], thereby allowing targeted release of these areas of fibrosis (Fig. 2). The iOCT further allows objective assessment of surgical success, being able to demonstrate formation of multiple cystic spaces within a rising bleb [15]. The iOCT also allows visualisation of the often concomitantly performed flap lift, which may be otherwise obscured by significant encapsulation and tenon's proliferation [22].

\section{Glaucoma drainage devices (GDD)}

GDDs allow the filtration of aqueous from the anterior chamber (AC) to an external reservoir, regulated by the formation of a fibrous capsule over the plate 4-6 weeks after surgery.

One of the challenges of GDD surgery is the accurate introduction of the tube into the AC. Traditionally a blind procedure, this step of the surgery can be challenging,

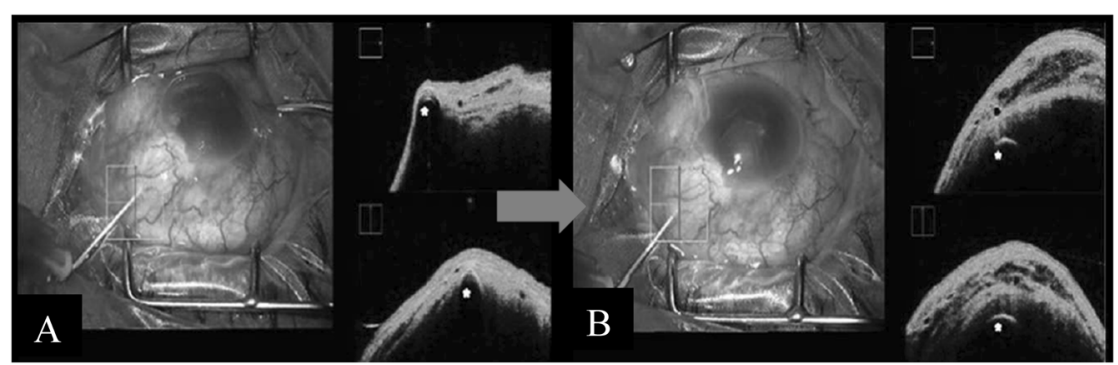

Fig. 2 Intra-operative and iOCT images in bleb needling surgery Courtesy of Dada et al. [15]. a Intra-operative clinical photograph presenting a flat vascularised bleb. The corresponding live iOCT imaging-horizontal scan (blue horizontal line) and vertical scan (red vertical line)_of the bleb shows apposition of the conjunctiva and sclera with minimal hyporeflective space, indicating a fibrosed/nonfiltering bleb with a $26 \mathrm{G}$ needle in situ (white asterisk). b iOCT imaging showing a relatively diffusely raised bleb with multiple hyporeflective spaces post bleb needling, with $26 \mathrm{G}$ needle in situ (white asterisk) 


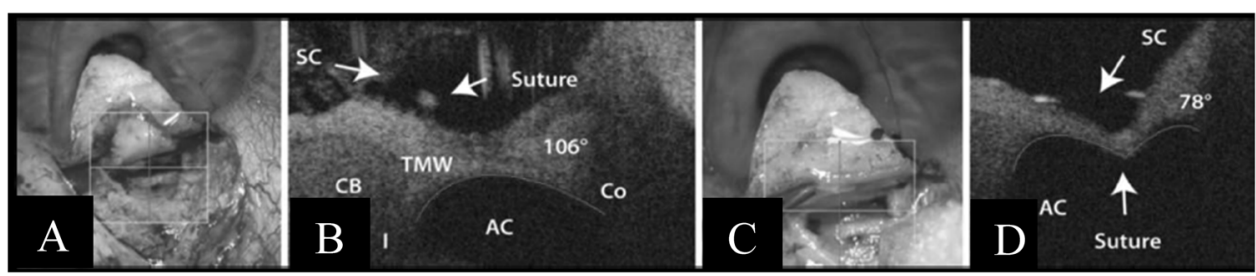

Fig. 3 Intra-operative and iOCT images in canaloplasty surgery Courtesy of Siebelmann et al. [27]. a Imaging through the prepared Descemet window before donning the suture. The cross shows the scanned area. b The vertical OCT scan showing the suture in the Schlemm's canal (SC), the trabecular meshwork (TMW), the ciliary

particularly in eyes with synechial angle closure and in conditions such as iridocorneal endothelial syndrome. Complications such as hyphema and peri-tube leak may occur with multiple, failed passes of the tube into the eye. Suboptimal placement of the tube may also cause long-term problems such as corneal decompensation [17, 37].

Swaminathan et al. [28] described the use of iOCT in guiding the tube into the eye during surgery, demonstrating its particular usefulness in the sulcus-placement of tubes. The direct visualisation of the tunnelling needle into the AC provides the surgeon certainty of its entry into the AC. Conversely, the anterior movement of the iris over the needle on iOCT indicates the entrance of the needle into the sulcus.

Improper placement and securing of the tube increases the risk [38] of tube erosion or extrusion of the GDD, a potentially serious complication [39] which increases the risk of endophthalmitis [38]. iOCT visualisation may aid in reducing the risk of tube erosion after GDD implantation by ensuring adequate coverage of conjunctiva and partialthickness sclera or donor tissue over the tube. The iOCT also has a valuable role in eyes with opaque corneas, with poor visualisation of the AC. It may also be used as a training tool for trainee surgeons [28].

\section{Minimally invasive glaucoma surgery (MIGS)}

Emerging data on the safety and efficacy of MIGS has been encouraging. The micro-incisional approach [26] adopted by MIGS has been associated with a higher safety profile, fewer complications and a more rapid recovery. However, MIGS involves more intricate surgical manoeuvring in areas which are poorly accessible or where visualisation is challenging-particularly for trabecular-bypass or anglebased MIGS.

Here, existing and potential applications of the iOCT, as an adjunct to improve the predictability and precision of MIGS, are discussed.

Canaloplasty is a procedure which utilises a microcatheter to expand the canal of Schlemm. Potential body $(\mathrm{CB})$, the iris $(\mathrm{I})$, the anterior chamber $(\mathrm{AC})$ and the cornea $(\mathrm{Co})$. The curvature of the chamber angle (red line) is convex. c Microscope image after closing the suture. The cross shows the scanned area. d The configuration of the chamber angle is now concave, with steepening of the angle between the SC and chamber angle

complications [14] include the failure to cannulate Schlemm's canal and Descemet membrane (DM) detachments. Siebelmann et al. [27] reported the use of iOCT as an adjunct to ab-externo canaloplasty surgery (Fig. 3). The iOCT allows the visualisation of intra-operative structures, facilitating precise microcatheter passage through the Schlemm's canal. The iOCT may also allow direct visualisation of the suture tightening against the Schlemm's canal -altering the shape of the AC, from a concave, to an Mshape. The appreciation of the degree between the AC and SC upon suture tightening may allow the surgeon to titrate the tension of the suture. Furthermore, the iOCT may help the surgeon minimise the risk of complications from this procedure by reducing the risk of complete penetration during dissection and minimising the incidence of DM detachments.

Trabecular aspiration is another form of angle-based MIGS. Its mechanism of action involves clearing the inter-trabecular spaces of extracellular debris [19], thereby improving aqueous drainage through the angle. iOCT-use would theoretically facilitate more accurate siting of the aspiration cannula. However, Heindl et al. [18] has reported the limitations of the iOCT in its application here-given the $840-\mathrm{nm}$ wavelength of the iOCT, visibility of the angle remains limited. In addition, the iOCT scans only vertically or horizontally and may not allow precise imaging of the aspirator tip in relation to the TM [18] (Fig. 4). OCT-guided trabecular aspiration using $1310 \mathrm{~nm}$ wavelengths has also been attempted in porcine eyes (Fig. 4).

The ab-interno trabectome is another angle-based MIGS device which may benefit from adjunct iOCT use, involving the removal of trabecular tissue to increase aqueous outflow [20]. The concurrent use of a gonioprism [29] or Swan-Jacobs gonioscopy lens [21] and the iOCT has been shown to aid the surgeon in visualising the angle and in identifying the opening of the inner wall of Schlemm's canal. Tilting the microscope and the iOCT from the regular $60^{\circ}$ to a more horizontal position may provide better visualisation for the surgeon [18]. 

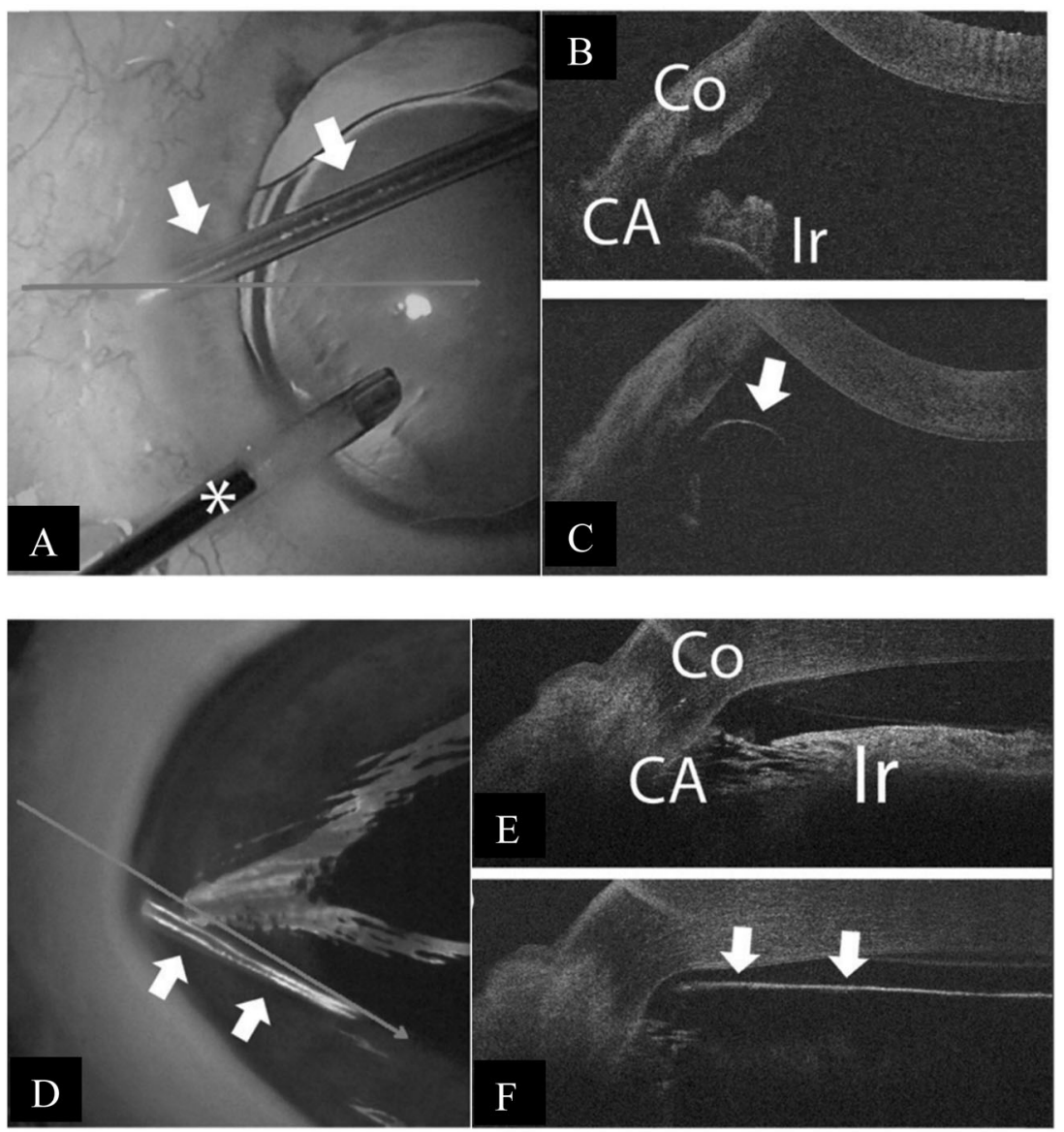

Fig. 4 Intra-operative and iOCT images during trabecular aspiration Courtesy of Heindl et al. [18]. a The aspiration cannula (arrows) is placed within the iridocorneal angle. To maintain intraocular pressure, an irrigation cannula is additionally placed within the anterior chamber (asterisk). The horizontal line demonstrates iOCT scanning direction. b iOCT image of the iridocorneal angle before placement of aspiration cannula. Iris and trabecular meshwork are shadowed by scleral tissue (Co cornea, CA chamber angle, Ir iris). $\mathbf{c}$ iOCT image of the aspiration cannula (arrow) within the iridocorneal angle. With a non-parallel scanning direction, the tip of the instrument is difficult to visualise. d Aspiration cannula (white arrows) is placed within the iridocorneal angle of the porcine eye. The long arrow demonstrates the iOCT scanning direction. e iOCT image of the iridocorneal angle before placement of aspiration cannula in porcine eye (Co cornea, CA chamber angle, Ir iris). f iOCT image with placement of aspiration cannula (arrows) within the iridocorneal angle in porcine eye. The relation between the cannula tip to the trabecular meshwork and the iris is clearly visible. However, structures behind the cannula are shadowed by the cannula

the XEN45 Gel Stent may also benefit from adjunct iOCT use. The XEN45 Gel Stent is made up of hydrophilic collagen-derived porcine gelatin. It provides a drainage shunt from the AC to the sub-conjunctival space [16] and theoretically elicits minimal extraocular fibrotic and vascular response [16].

The iOCT may be useful during XEN45 implantation as it may help determine the final placement of the implant during surgery. The location of optimal placement of the XEN45 implant is a subject of debate [16]. However, regardless of the space targeted, there is inadequate visualisation past the Tenon's when targeting the sub-Tenon's space, and there is a risk of conjunctival perforation when targeting the superficial sub-conjunctival space. The use of iOCT may decrease intra-operative risks and optimise 

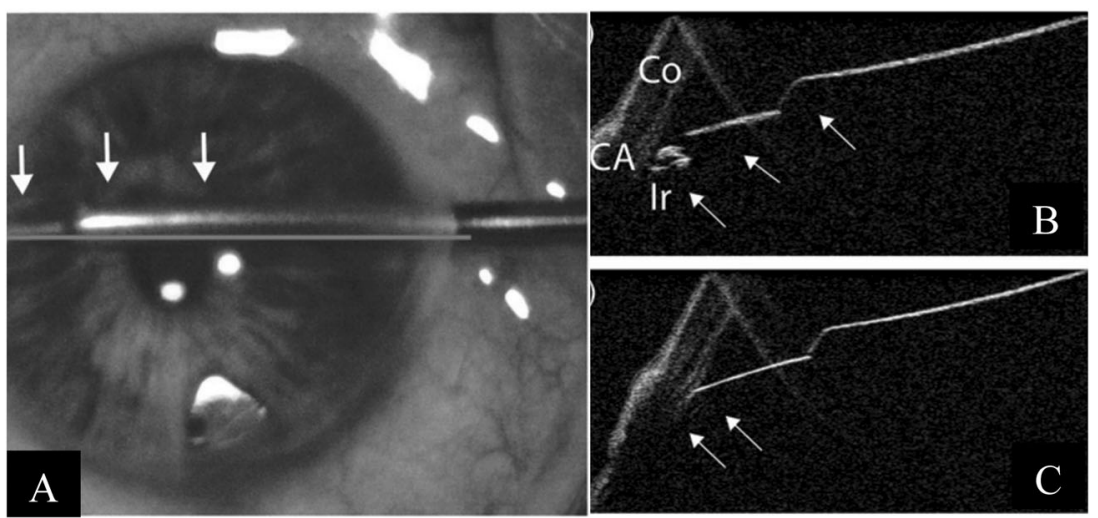

Fig. 5 Intra-operative and iOCT images during ab-interno trabeculotomy Courtesy of Heindl et al. [18]. a The trabectome (arrows) is placed within the iridocorneal angle. The horizontal line demonstrates the iOCT scanning direction. $\mathbf{b}$ iOCT image of the iridocorneal angle during placement of the trabectome. Trabecular meshwork is shadowed by scleral tissue, but iris is visible (Co cornea, CA chamber angle, Ir iris). c iOCT section of the trabectome (arrows) within the iridocorneal angle. Iris is not visualised due to the shadowing by the trabectome
Fig. 6 Intra-operative images of sub-conjunctival haemorrhage during XEN implantation Courtesy of Leonard W. L. Yip, FRCSEd and Bryan C. H. Ang, FRCOphth, Singapore.

a, b Development of subconjunctival haemorrhage during XEN implantation obscuring view of emerging stent. c Good visualisation of the XEN gel stent at the beginning of a needling procedure. $\mathbf{d}$ Poor visualisation of the XEN gel stent as needling

progresses, due to subconjunctival haemorrhage and rising of the bleb. e Ab-externo XEN implantation in an eye undergoing open revision after prior XEN implantation, as the previous stent was found not be patent during surgery
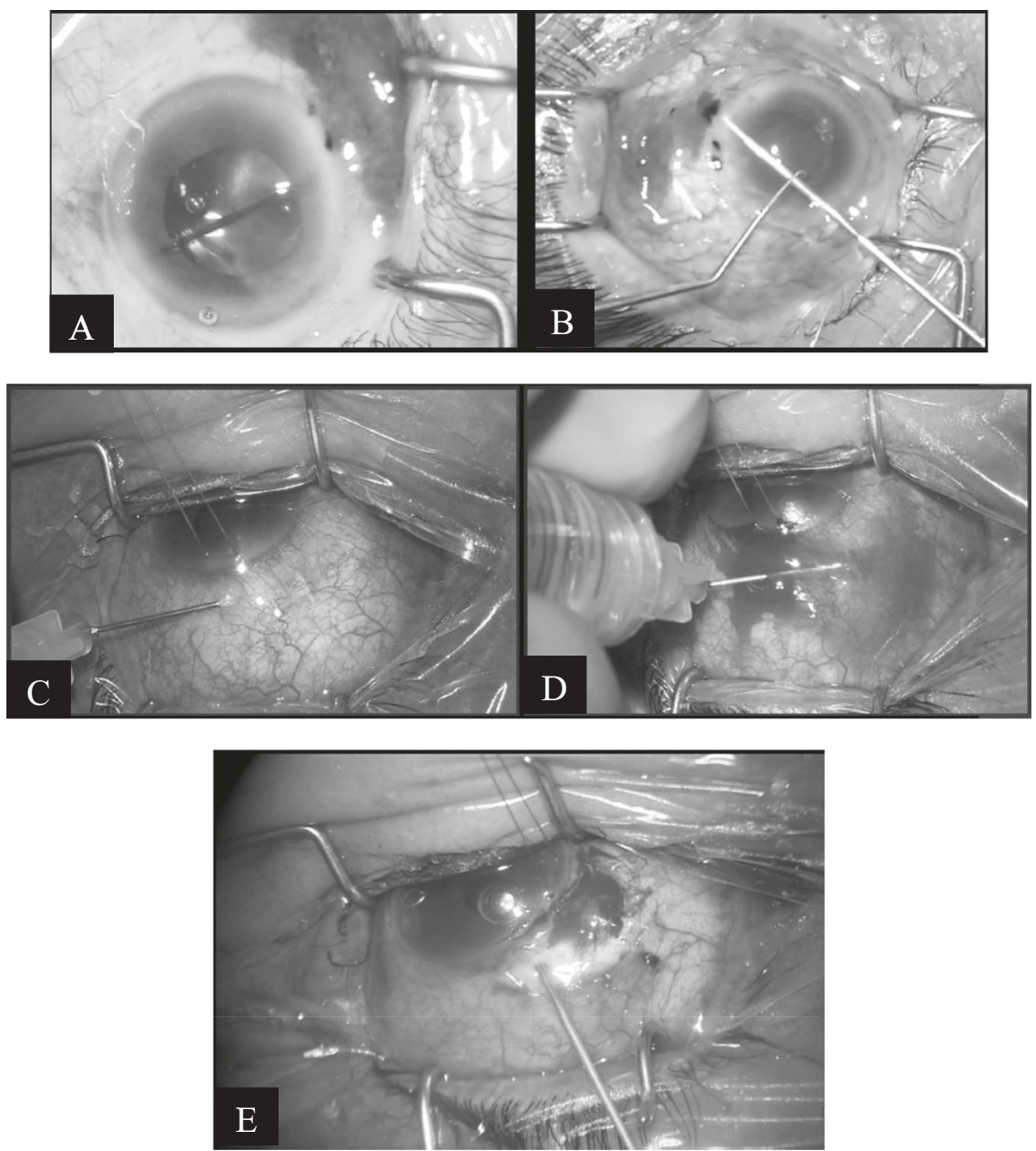

placement of the implant. The iOCT may also be useful in visualising the XEN45 implant in the event of subconjunctival haemorrhage, which obscures the surgical field and hinders accurate placement of the implant, at times requiring the surgeon to abort the procedure entirely (Fig. 6).

Post-operatively, the OCT would aid in the needling of the XEN bleb. While similar to its application in 
post-trabeculectomy bleb needling, the benefits of the iOCT in needling of the XEN bleb may be more pronounced, given the need for the surgeon to avoid inadvertently transecting the XEN stent during the needling procedure. This becomes more necessary in XEN blebs with thick Tenon's proliferation which may entirely obscure the stent, or when sub-conjunctival haemorrhage occurs during the needling procedure (Fig. 6).

Finally, iOCT would be useful in the ab-externo implantation of the XEN implant, similar to its application during scleral tunnelling in GDD implantation. This may be in the context of primary trans-conjunctival implantation or secondary implantation during open revision of a scarred XEN bleb when the patency of the in-situ XEN is suspect.

\section{Limitations of iOCT}

Despite its usefulness and potential applications in glaucoma surgery, the iOCT is not without its limitations.

Firstly, as both stand-alone iOCTs and microscopeintegrated iOCT systems are not inexpensive, cost and accessibility remain a limitation in the use of this technology. Secondly, adopting iOCT in one's surgical practice may involve a steep initial learning curve. The microscopeintegrated iOCT platforms require simultaneous viewing of both the surgical field and the OCT image during surgery, through both oculars of the microscope. Thirdly, the scanning raster of current $\mathrm{iOCT}$ platforms has a restricted scanning area and this may require the surgeon to constantly move the scanning zone during surgery to visualise the instrument tip or area of interest. Fourthly, optimal iOCT images may be difficult to obtain. Current iOCT platforms do not respond well to movements of the eye during surgery-motion artefacts are generated, and there is a noticeable time lag in between real-time movement and the displaying of images. A dry corneal surface, incorrect magnification and improper focus may also compromise image quality. Fifthly, structures posterior to metallic instruments may not be able to be visualised due to impedance of OCT light source wavelength [33].

\section{Future research and developments in iOCT technology}

Despite the existing limitations, iOCT technology continues to progress rapidly since it was first introduced into clinical practice.

Firstly, advancements in iOCT technology continue to optimise surgeon feedback. This is achieved through the use of HUD and OCT visualisation. The use of microdisplays project live, 2D-OCT images into one or both oculars of the operating microscope [8, 31, 40-42]. As
HUD technology continues to evolve and its potential in providing real-time iOCT feedback expands, the iOCT may see greater adoption in surgical practice.

Secondly, there have been advances in software system development and the designing of automated iOCT algorithms, which are already available for DSAEK [43] and macular hole [44] procedures. In addition, the use of automated stereo-vision tracking in iOCT-guided surgeries [4547] is being explored as the currently limited acquisition rates of the iOCT may not be able to entirely cope with the complexity of the three-dimensional visualisation methods required in such surgeries [48]. Experimental technology has demonstrated the technical feasibility of surgical instrument tip-tracking and corroboration between the iOCT image and the operating field [48].

Thirdly, new material has been used for surgical instruments to overcome the difficulties associated with light scattering and the shadowing characteristics of metal instruments [8, 13, 24, 49]. New instruments incorporating semi-transparent material from its tip may allow for transmission of OCT signals, enabling visualisation of tissues deep to these instruments [33]. Advancements in software systems for iOCT analysis may also be able to compensate for the scattering caused by metallic surgical instruments [50].

Fourthly, swept-source OCT (SS-OCT) holds great potential in replacing current iterations of iOCT systems. Since 2012, the SS-OCT has been made available in clinical practice, with its superior visualisation capabilities [51] allowing visualisation of the choroid, vitreous and retinal structures behind dense pre-retinal haemorrhages, due to its deeper penetration and faster acquisition time [51, 52]. Surgical steps that may benefit from enhanced visualisation with the SS-OCT include scleral tunnel creation, tube shunt positioning and suturing, scleral flap creation, sclerotomy and iridectomy [53]. It also allows for faster imaging across a larger imaging range, enabling the real-time evaluation of instruments in motion during surgical manoeuvres [24, 36, 54]. Pasricha et al. [24] has reported the use of the swept-source microscope-integrated OCT (SS-MIOCT) to enhance visualisation in glaucoma surgeries. The SSMIOCT is capable of live, four-dimensional (three-dimensional across time) imaging. This overcomes the 'time-lag' observed in 2D SD-MIOCT systems and is able to respond real-time during surgery. SS-MIOCT is postulated to be more useful than conventional iOCT in patients with opaque cornea, thin sclera, shallow ACs, PAS formation, and eyes in which visualisation of the position of the lens is difficult [53]. In high myopes, with at times, a wider and more posterior anatomic limbus, the use of SS-iOCT may help reduce the risk of improper insertion of tube [24]. The use of the SS-MIOCT in scleral tunnelling is depicted in Fig. 7. 

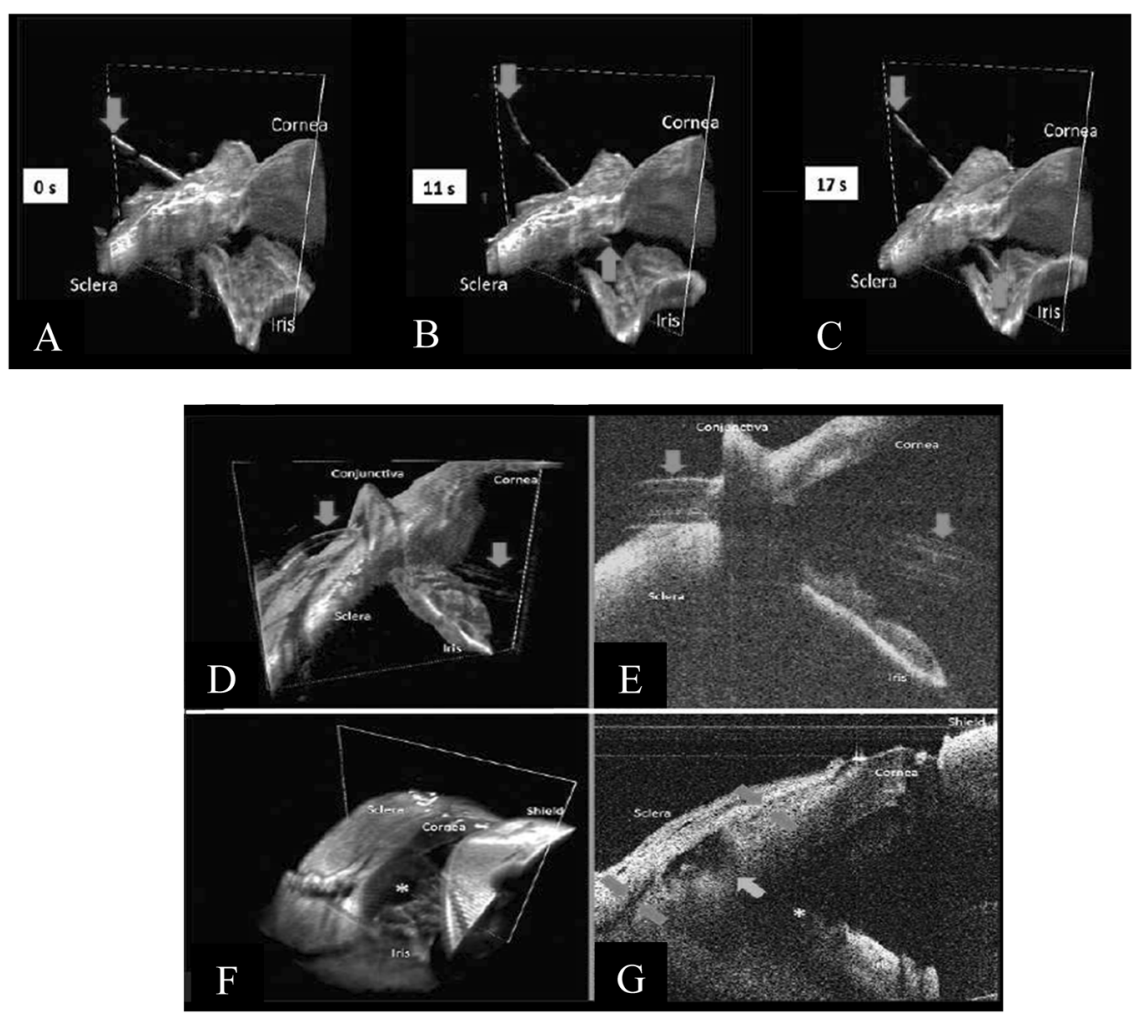

Fig. 7 SS-MIOCT demonstrating scleral tunnelling in live 4D (threedimensional across time) Courtesy of Pasricha et al. [24]. a Scleral tunnelling using a 23-gauge needle (red arrows) advancing from the surface of the sclera under direct visualisation, preventing the creation of peripheral anterior synechiae formation post-operatively (b) Through the scleral into the anterior chamber. $\mathbf{c}$ Through to the deepest point in the anterior chamber, creating a scleral tunnel for subsequent tube shunt placement. d, e Tube shunt (red arrows) insertion through

\section{Conclusion}

iOCT use in ophthalmic surgery is becoming increasingly prevalent and has already been applied in various surgeries and procedures in the field of glaucoma. With the greater adoption of MIGS, iOCT may further contribute in facilitating surgical techniques and improving outcomes. While iOCT offers many advantages, there are still limitations to be overcome and iOCT technology continues to evolve to optimise imaging quality and userexperience.

\section{Compliance with ethical standards}

Conflict of interest BCHA has previously received funding from Glaukos Corporation (travel, research), Santen Pharmaceutical Co., Ltd. (travel, consultant) and Allergan plc (travel, research, consultant). $\mathrm{He}$ has not received funding for his work in this publication. SD has consulted for New World Medical and received funding (travel, research). He has not received funding for his work in this publication. The remaining author declares that he has no conflict of interest. the previously established scleral tunnel into the anterior chamber, with proper positioning anterior to the iris and without corneal touch (SS-MIOCT three-dimensional volume (D) with a white box demarcating its corresponding two-dimensional B-scan (E)). f, g Scleral flap interface (green arrows), sclerotomy (blue arrow), and iridectomy (yellow asterisk) at the end of the trabeculectomy surgery (SS-MIOCT three-dimensional volume $(\mathrm{F})$ with a white box demarcating its corresponding two-dimensional B-scan $(\mathrm{G})$ )

Publisher's note Springer Nature remains neutral with regard to jurisdictional claims in published maps and institutional affiliations.

\section{References}

1. Swanson EA, Huang D. Ophthalmic OCT reaches $\$ 1$ billion per year. Retinal Physician. 2011;53:58-9.

2. Eguchi H, Kusaka S, Arimura-Koike E, Tachibana K, Tsujioka D, Fukuda M, et al. Intraoperative optical coherence tomography (RESCAN ${ }^{\circledR} 700$ ) for detecting iris incarceration and iridocorneal adhesion during keratoplasty. Int Ophthalmol. 2017;37:761-5.

3. Juthani VV, Goshe JM, Srivastava SK, Ehlers JP. Association between transient interface fluid on intraoperative OCT and textural interface opacity after DSAEK surgery in the PIONEER study. Cornea. 2014;33:887-92.

4. Saad A, Guilbert E, Grise-Dulac A, Sabatier P, Gatinel D. Intraoperative OCT-assisted DMEK: 14 consecutive cases. Cornea 2015;34:802-7.

5. Cost B, Goshe JM, Srivastava S, Ehlers JP. Intraoperative optical coherence tomography-assisted descemet membrane endothelial keratoplasty in the DISCOVER study. Am J Ophthalmol. 2015; 160:430-7. 
6. Scorcia V, Busin M, Lucisano A, Beltz J, Carta A, Scorcia G. Anterior segment optical coherence tomography guided bigbubble technique. Ophthalmology. 2013;120:471-6.

7. Ehlers JP, Dupps WJ, Kaiser PK, Goshe J, Singh RP, Petkovsek D, et al. The prospective intraoperative and perioperative ophthalmic imaging with optical coherence tomography (PIONEER) study: 2-year results. Am J Ophthalmol. 2014;158: 999-1007. el

8. Ehlers JP, Goshe J, Dupps WJ, Kaiser PK, Singh RP, Gans R, et al. Determination of feasibility and utility of microscope-integrated optical coherence tomography during ophthalmic surgery: the DISCOVER Study RESCAN Results. JAMA Ophthalmol. 2015; 133:1124-32.

9. Au J, Goshe J, Dupps WJ Jr., Srivastava SK, Ehlers JP. Intraoperative optical coherence tomography for enhanced depth visualization in deep anterior lamellar keratoplasty From the PIONEER study. Cornea. 2015;34:1039-43.

10. AlTaan SL, Termote K, Elalfy MS, Hogan E, Werkmeister R, Schmetterer L, et al. Optical coherence tomography characteristics of different types of big bubbles seen in deep anterior lamellar keratoplasty by the big bubble technique. Eye. 2016;30:1509-16.

11. Hirnschall N, Amir-Asgari S, Maedel S, Findl O. Predicting the postoperative intraocular lens position using continuous intraoperative optical coherence tomography measurements. Investig Ophthalmol Vis Sci. 2013;54:5196-203.

12. Das S, Kummelil MK, Kharbanda V, Arora V, Nagappa S, Shetty $\mathrm{R}$, et al. Microscope integrated intraoperative spectral domain optical coherence tomography for cataract surgery: uses and applications. Curr Eye Res. 2016;41:643-52.

13. Binder S, Falkner-Radler CI, Hauger C, Matz H, Glittenberg C. Feasibility of intrasurgical spectral-domain optical coherence tomography. Retina. 2011;31:1332-6.

14. Bull H, von Wolff K, Körber N, Tetz M. Three-year canaloplasty outcomes for the treatment of open-angle glaucoma: European study results. Graefe's Arch Clin Exp Ophthalmol. 2011;249:1537-45.

15. Dada T, Angmo D, Midha N, Sidhu T. Intraoperative optical coherence tomography guided bleb needling. $\mathrm{J}$ ophthalmic Vis Res. 2016;11:452-4.

16. De Gregorio A, Pedrotti E, Stevan G, Bertoncello A, Morselli S. XEN glaucoma treatment system in the management of refractory glaucomas: a short review on trial data and potential role in clinical practice. Clin Ophthalmol. 2018;12:773-82.

17. Giovingo M. Complications of glaucoma drainage device surgery: a review. Semin Ophthalmol. 2014;29:397-402.

18. Heindl LM, Siebelmann S, Dietlein T, Hüttmann G, Lankenau E, Cursiefen C, et al. Future prospects: assessment of intraoperative optical coherence tomography in ab interno glaucoma surgery. Curr Eye Res. 2015;40:1288-91.

19. Jacobi PC, Dietlein TS, Krieglstein GK. Perspectives in trabecular surgery. Eye 2000;14:519.

20. Jordan JF, Wecker T, van Oterendorp C, Anton A, Reinhard T, Boehringer D, et al. Trabectome surgery for primary and secondary open angle glaucomas. Graefe's Arch Clin Exp Ophthalmol. 2013;251:2753-60.

21. Junker B, Jordan JF, Framme C, Pielen A. Intraoperative optical coherence tomography and ab interno trabecular meshwork surgery with the Trabectome. Clin Ophthalmol. 2017;11:1755-60.

22. Kumar RS, Jariwala MU, V SA, Venugopal JP, Puttaiah NK, Balu $\mathrm{R}$, et al. A pilot study on feasibility and effectiveness of intraoperative spectral-domain optical coherence tomography in glaucoma procedures. Transl Vis Sci Technol. 2015;4:2.

23. Li X, Wei L, Dong X, Huang P, Zhang C, He Y, et al. Microscope-integrated optical coherence tomography for imageaided positioning of glaucoma surgery. SPIE. 2015;20:76001.

24. Pasricha ND, Bhullar PK, Shieh C, Viehland C, Carrasco-Zevallos OM, Keller B, et al. Four-dimensional microscope-integrated optical coherence tomography to enhance visualization in glaucoma surgeries. Indian J Ophthalmol. 2017;65:57-9.

25. Mastropasqua R, Fasanella V, Agnifili L, Curcio C, Ciancaglini M, Mastropasqua L. Anterior segment optical coherence tomography imaging of conjunctival filtering blebs after glaucoma surgery. BioMed Res Int. 2014;2014:610623.

26. Saheb H, Ahmed IIK. Micro-invasive glaucoma surgery: current perspectives and future directions. Curr Opin Ophthalmol. 2012; 23:96-104.

27. Siebelmann S, Cursiefen C, Lappas A, Dietlein T. Intraoperative optical coherence tomography enables noncontact imaging during canaloplasty. J Glaucoma. 2016;25:236-8.

28. Swaminathan SS, Chang TC. Use of intraoperative optical coherence tomography for tube positioning in glaucoma surgery. JAMA Ophthalmol. 2017;135:1438-9.

29. Tanito M. Optical coherence tomography observation of gonio structures during microhook ab interno trabeculotomy. J Ophthalmol 2017;2017:4.

30. Moher D, Liberati A, Tetzlaff J, Altman DG. Preferred reporting items for systematic reviews and meta-analyses: the PRISMA statement. J Clin Epidemiol. 2009;62:1006-12.

31. Palácios RM, de Carvalho ACM, Maia M, Caiado RR, Camilo DAG, Farah ME. An experimental and clinical study on the initial experiences of Brazilian vitreoretinal surgeons with heads-up surgery. Graefe's Arch Clin Exp Ophthalmol. 2019;257:473-83.

32. Geerling G, Müller M, Winter C, Hoerauf H, Oelckers S, Laqua $\mathrm{H}$, et al. Intraoperative 2-dimensional optical coherence tomography as a new tool for anterior segment surgery. Arch Ophthalmol. 2005;123:253-7.

33. Ehlers JP, Uchida A, Srivastava SK. Intraoperative optical coherence tomography-compatible surgical instruments for real-time imageguided ophthalmic surgery. Br J Ophthalmol. 2017;101:1306-8.

34. Samsudin A, Eames I, Brocchini S, Khaw PT. The influence of scleral flap thickness, shape, and sutures on intraocular pressure (IOP) and aqueous humor flow direction in a trabeculectomy model. J Glaucoma. 2016;25:e704-12.

35. Tanaka D, Nakanishi H, Hangai M, Akagi T, Morooka S, Ikeda $\mathrm{HO}$, et al. Influence of high myopia on outcomes of trabeculectomy with mitomycin $\mathrm{C}$ in patients with primary open-angle glaucoma. Jpn J Ophthalmol. 2016;60:446-53.

36. McKee H, Ye C, Yu M, Liu S, Lam DSC, Leung CKS. Anterior chamber angle imaging with swept-source optical coherence tomography: detecting the Scleral Spur, Schwalbe's Line, and Schlemm's Canal. J Glaucoma. 2013;22:468-72.

37. Zalloum JN, Ahuja RM, Shin D, Weiss JS. Assessment of corneal decompensation in eyes having undergone molteno shunt procedures compared to eyes having undergone trabeculectomy. CLAO J 1999;25:57-60.

38. Al-Torbak AA, Al-Shahwan S, Al-Jadaan I, Al-Hommadi A, Edward DP. Endophthalmitis associated with the Ahmed glaucoma valve implant. Br J Ophthalmol. 2005;89:454-8.

39. Bailey AK, Sarkisian SR. Complications of tube implants and their management. Curr Opin Ophthalmol. 2014;25:148-53.

40. Ehlers JP, Srivastava SK, Feiler D, Noonan AI, Rollins AM, Tao YK. Integrative advances for OCT-guided ophthalmic surgery and intraoperative OCT: microscope integration, surgical instrumentation, and heads-up display surgeon feedback. PloS ONE. 2014;9:e105224.

41. Siebelmann S, Steven P, Hos D, Hüttmann G, Lankenau E, Bachmann B, et al. Advantages of microscope-integrated intraoperative online optical coherence tomography: usage in Boston keratoprosthesis type I surgery. SPIE. 2016;21:016005.

42. Pfau M, Michels S, Binder S, Becker MD. Clinical experience with the first commercially available intraoperative optical coherence tomography system. Ophthalmic Surg Lasers Imaging Retina. 2015;46:1001-8. 
43. Hallahan KM, Cost B, Goshe JM, Dupps WJ Jr., Srivastava SK, Ehlers JP. Intraoperative Interface fluid dynamics and clinical outcomes for intraoperative optical coherence tomographyassisted descemet stripping automated endothelial keratoplasty from the PIONEER study. Am J Ophthalmol. 2017;173:16-22.

44. Xu D, Yuan A, Kaiser PK, Srivastava SK, Singh RP, Sears JE, et al. A novel segmentation algorithm for volumetric analysis of macular hole boundaries identified with optical coherence tomographyvolumetric macular hole segmentation. Investig Ophthalmol Vis Sci. 2013;54:163-9.

45. Izatt J, Chiu S, Farsiu S, Hahn P, Migacz J, Toth C. Inventors of surgical tool tracking feedback control system for intraoperative optical coherence tomography. United States of America: Duke University Office of Licensing and Ventures.

46. Sharma N, Urkude J, Chaniyara M, Titiyal JS. Microscopeintegrated intraoperative optical coherence tomography-guided small-incision lenticule extraction: new surgical technique. J Cataract Refractive Surg. 2017;43:1245-50.

47. Hahn P, Carrasco-Zevallos O, Cunefare D, Migacz J, Farsiu S, Izatt JA, et al. Intrasurgical human retinal imaging with manual instrument tracking using a microscope-integrated spectraldomain optical coherence tomography device. Transl Vis Sci Technol 2015;4:1.

48. El-Haddad MT, Tao YK. Automated stereo vision instrument tracking for intraoperative OCT guided anterior segment ophthalmic surgical maneuvers. Biomed Opt Express. 2015;6: 3014-31.

49. Ehlers JP, Tao YK, Farsiu S, Maldonado R, Izatt JA, Toth CA. Integration of a spectral domain optical coherence tomography system into a surgical microscope for intraoperative imaging. Investigative Ophthalmol Vis Sci. 2011;52:3153-9.

50. Ehlers JP, Tao YK, Farsiu S, Maldonado R, Izatt JA, Toth CA. Visualization of real-time intraoperative maneuvers with a microscope-mounted spectral domain optical coherence tomography system. Retina. 2013;33:232-6.

51. Kishi S. Impact of swept source optical coherence tomography on ophthalmology. Taiwan J Ophthalmol. 2016;6:58-68.

52. Miller AR, Roisman L, Zhang Q, Zheng F, Rafael de Oliveira Dias J, Yehoshua Z, et al. Comparison between spectral-domain and swept-source optical coherence tomography angiographic imaging of choroidal neovascularization imaging of CNV with SS-OCTA and SD-OCTA. Investig Ophthalmol Vis Sci. 2017; 58:1499-505.

53. Pasricha ND, Shieh C, Carrasco-Zevallos OM, Keller B, Izatt JA, Toth CA, et al. Real-time microscope-integrated OCT to improve visualization in DSAEK for advanced bullous keratopathy. Cornea. 2015;34:1606-10.

54. McNabb RP, Challa P, Kuo AN, Izatt JA. Complete $360^{\circ}$ circumferential gonioscopic optical coherence tomography imaging of the iridocorneal angle. Biomed Opt Express. 2015;6:1376-91. 